\title{
Nurse practitioner oder Medizinische Praxiskoordinatorin?
}

\section{Emil Schalch}

Präsident OdA Berufsbildung MPA
Korrespondenz:

Dr. med. Emil Schalch

Fischmattstrasse 2

CH-6315 Oberägeri

emil.schalch(at)hin.ch
Im Herbst 2007 veröffentlichte eine Arbeitsgruppe der Schweizerischen Akademie der Medizinischen Wissenschaften SAMW den Bericht «Die zukünftigen Berufsbilder von Ärztinnen/Ärzten und Pflegenden in der ambulanten und klinischen Praxis [1]. In diesem Bericht wurden Möglichkeiten untersucht, wie durch neue Berufsbilder im Schweizerischen Gesundheitswesen Herausforderungen wie Personalmangel und ungleiche Personalverteilung, steigende Kosten der Gesundheitsversorgung und der Ruf nach höherer Kostenwirksamkeit und Qualitätssteigerung einschliesslich der beruflichen Weiterentwicklung und des Wunsches nach einem besseren Arbeitsleben bewältigt werden könnten. Im Fokus der Überlegungen standen dabei die Berufe der Ärzte und der Pflegenden. Kürzlich hat die SAMW nun eine Broschüre veröffentlicht, die den damaligen Bericht mit einem kritischen Kommentar ergänzt und feststellt, dass die Schweiz auf dem damals vorgeschlagenen Weg einer weiteren Akademisierung der Pflegeberufe und der Übernahme von klassischen ärztlichen Tätigkeiten durch Pflegefachleute («Nurse practitioner») nicht viel weitergekommen ist. In der Analyse dieser Tatsache fragt sich die Arbeitsgruppe, «ob Entwicklungen nahezu epochalen Charakters im Sinne eines immer mächtiger werdenden Trends einfach sgeschehen oder ob sie eingeleitet, gefördert, beschleunigt oder gesteuert werden können - und durch wen? Sind es Berufsverbände, ist es der Staat, sind es hellhörige Wissenschaftler oder einzelne Pioniere und Pionierorganisationen, die aktiv werden müssen?» [1, S. 27].

Die Antwort findet sich in einem äusserst lesenswerten Grundsatzpapier, erarbeitet für die Europäische Ministerkonferenz der WHO zum Thema Gesundheitssysteme im Juni 2008 in Tallinn, Estland. Bourgeault et al. untersuchten darin den Umgang der Gesundheitssysteme Englands, Deutschlands, Spaniens, Finnlands und der Russischen Föderation mit deren Personalengpässen in der ärztlichen Grundversorgung [2]. Unter der Überschrift «Zentrale Aussagen» stehen darin folgende, wahrscheinlich auch für die Schweiz gültige Sätze:

«Initiativen für einen optimalen Qualifikationsmix müssen vom Bedarf geleitet sein. Ein sensibles Vorgehen innerhalb des Gesundheitssystems und gegenüber dem Gesundheitspersonal ist ratsam. Das Überstülpen einer vorgefertigten Lösung hilft nicht weiter.»

«Für jede dieser Initiativen ist eine Unterstützung durch die betroffenen Fachorganisationen und die Regierung unverzichtbar.»

\section{«Nurse practitioner» ou coordinatrice médicale?}

La plupart des pays européens sont déjà ou seront confrontés à l'avenir à de réels problèmes de pénurie de spécialistes en médecine de premier recours. Pour les résoudre, il s'agit de procéder à des changements dans le recoupement des qualifications professionnelles entre les médecins et les autres professionnels de la santé impliqués dans la prise en charge des patients.

$\mathrm{Au}$ vu des situations locales respectives [2] au moment de la création de nouveaux profils professionnels, ces pays sont plus ou moins avancés dans leur manière d'appréhender la médecine de premier recours pour répondre aux défis futurs et aux problèmes de personnel qui y sont liés. Dans notre pays, les efforts engagés pour introduire le métier de «nurse practitioner» dans le but de seconder les médecins font du surplace - et cela n'a rien d'étonnant [1]. Les généralistes n'approuvent pas cette solution, car ils estiment qu'elle n'est pas compatible avec notre système de soins. La comparaison entre les «nurse practitioners» et les assistantes médicales au bénéfice d'un certificat fédéral de capacité et d'une formation complémentaire de coordinatrice médicale montre que I'on dispose d'une solution viable pour pallier au manque de personnel en médecine de premier recours. Cette option n'est certes pas gratuite, mais elle s'avère quand même moins onéreuse sur les plans financier et politique que la solution prisée et tant vantée de la «nurse practitioner». 
In ihrem Kommentar zum Bericht hinterfragt erfreulicherweise auch die Arbeitsgruppe der SAMW ihren ursprünglichen Blickwinkel: «Um eine umfassendere und 〈vollständigere〉 Perspektive auf die Gesundheitsberufe zu haben, ist zu überlegen, ob ein nächster Bericht ins Auge zu fassen wäre, der den Fokus auf andere «Gesundheitsberufe legt [1, S. 25].

Eine Erweiterung des Blickwinkels im Hinblick auf Personalmangel und neue Berufsbilder ist aus zwei Gründen dringend nötig

- Es «muss bedacht werden, dass es kaum Sinn macht, einen Mangelberuf (Ärzte) durch einen anderen Mangelberuf (Pflegefachleute) zu substituieren» $[1$, S. 30].

- Wichtige Entwicklungen gerade im Personalbereich der Grundversorgung können erst dann wahrgenommen werden, wenn die akademische Brille, welche nur die Verbindung Arzt-Pflegefachperson erkennt, endlich abgelegt wird. Dieses Ablegen müsste zur Erkenntnis führen, dass es in der hausärztlichen Grundversorgung traditionell einen anderen Dualismus gibt, welcher sich täglich bewährt: die Verbindung Hausarzt-Medizinische Praxisassistentin.
Die Idee, die Personalprobleme der Grundversorgung in der Grundversorgung selbst mit Personalressourcen der Grundversorgung zu lösen, drängt sich somit auf!

Weil die Fachgesellschaften «nicht darum herumkommen», «die notwendigen Massnahmen einzuleiten» [1, S. 29], haben einige Hausärzte und Medizinische Praxisassistentinnen im Rahmen der OdA Berufsbildung MPA mit Unterstützung von Hausärzte Schweiz, der FMH, der im «Futuro»-Projekt zusammengeschlossenen Managed-Care-Organisationen der Deutschschweiz, des Schweizerischen Verbandes der Medizinischen Praxisassistentinnen SVA und des Institutes für Hausarztmedizin der Universität Zürich eine alltagstaugliche Alternative zur Nurse practitioner erarbeitet.

Im Folgenden soll nicht im Detail auf das Weiterbildungsprojekt «Berufsprüfung MPA» eingegangen werden (dies wird an anderer Stelle geschehen), sondern im direkten Vergleich aufgezeigt werden, dass es sich lohnt, die «Medizinische Praxiskoordinatorin» in die Überlegungen im Zusammenhang mit dem Erhalt einer qualitativ hochstehenden Grundversorgung in unserem Lande einzubeziehen.

\section{Tabelle 1}

Unterschiede des Berufsbildes «Nurse practitioner» und «Medizinische Praxiskoordinatorin» auf einen Blick.

\section{Ziel \\ Mittel \\ Projekt \\ Vorbildung \\ Geplante Ausbildung}

\section{Ausbildungsfinanzierung}

Auftrag

\section{Gesetzesänderungen}

Einbindung/Anbindung

Infrastrukturkosten

Entschädigung der Leistungen

Folgen des Abrechnungsmodus

Wirkung des Projektes auf den angestammten Arbeitsplatz

\section{Fazit}

\section{Nurse practitioner}

Entlastung/Ersatz der Hausärzte

Kompetenzenteilung

Top-down

Pflege

Tertiärstufe, Fachhochschule, Master-Titel Umfassende, jedoch theorielastige Ausbildung,

\section{Steuerzahler?}

Schwammig: Ersatz des Hausarztes in «bestimmten» Bereichen, Stellen von «bestimmten» Diagnosen, Ausstellen von Rezepten

\section{nötig}

Selbständig. Anbindung ans Spital?

Unklar. Wo ist die NP angebunden? Im Spital? Eigene Praxis?

Über KK selbständig abrechenbare Eigenleistungen

Falls der Gesundheitskuchen in Zukunft für Leistungserbringer weiterhin gleich gross bleiben soll, konkurrenziert die NP den Hausarzt.

Abwanderung aus dem Spital

Verstärkung des Mangels an Pflegefachfrauen im Spital

Projekt sicher machbar, politisch jedoch teuer, weil unser Gesundheitssystem durch staatliche Eingriffe an das Projekt NP angepasst werden müsste. Dieses konkurrenziert die Hausärzte. Widerstand der Ärzteschaft ist somit absehbar.

\section{Medizinische Praxiskoordinatorin}

Entlastung der Hausärzte

Kompetenzenteilung

Bottom-up

\section{Grundversorgung}

Tertiärstufe Berufsbildung, Eidgen. Fachausweis Auf den vorgesehenen Einsatz zugeschnittene, duale «hands on»-Ausbildung

\section{Ärzteschaft/MPA}

Klar: unter der Verantwortung des Hausarztes Koordination der Versorgung und selbständige Führung von Patienten mit chronischen Krankheiten unnötig

Einbindung in die Grundversorgerpraxis

Unwesentlich, da Arbeitsplatz in der Grundversorgerpraxis schon vorhanden

Praxiskoordinatorin bezieht Lohn. Praxis rechnet die erbrachten CCM-Leistungen mit der Kasse gemäss Vertrag ab.

Abrechenbare, durch die Praxiskoordinatorin erbrachte Leistungen schaffen mehr ökonomische und organisatorische Freiheiten in der Grundversorgerpraxis und verbessern die Arbeitsbedingungen.

Attraktivitätssteigerung des Arbeitsplatzes Hausarztpraxis; Aufstiegsmöglichkeiten für MPA wirken der Abwanderung aus dem Beruf entgegen.

Projekt empfiehlt sich durch seine Grundversorgernähe und durch die nahtlose Einpassung in die Hausarztpraxis. Es entspricht sowohl dem Bedürfnis der MPA und der Hausärzte als auch dem der Bevölkerung. 


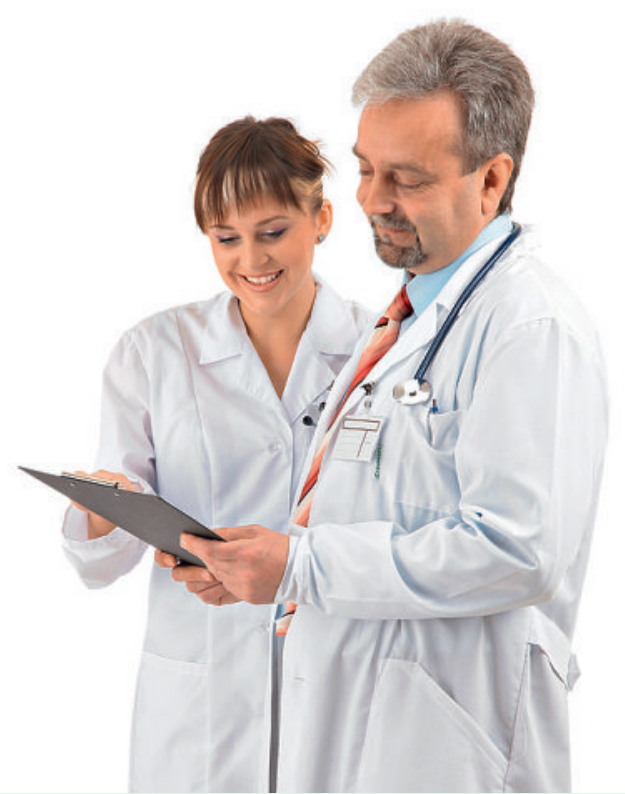

Für die Übertragung weiterer Aufgaben an die MPA ist eine Ausbildung als Medizinische Praxiskoordinatorin mit eidgenössischem Fachausweis erforderlich.

\section{Kann eine MPA wirklich den Hausarzt ersetzen?}

Diese Frage, die man immer wieder hört, stellt sich eigentlich gar nicht. Es gibt gar keinen Grund, den Hausarzt zu ersetzen. Im Gegenteil: Die Bevölkerung der Schweiz möchte ihren Hausarzt bzw. ihre Hausärztin behalten, und die Hausärzte kämpfen nicht für ihre Ersetzung oder Entlastung, sondern lediglich für die Rückgewinnung ihrer durch restriktive Auslegung des TARMEDs und durch zusätzlich übertragene Managed-Care-Aufgaben abhanden gekommene ökonomische, berufliche und private Freiheit. Es geht folglich lediglich darum, der Hausarztpraxis die Kompetenz zu geben, auch im abrechenbaren (klinischen) Bereich die Arbeit im Team so aufteilen zu dürfen, dass die Leistungen der Praxis wieder wirksam, zweckmässig und wirtschaftlich erbracht werden können nicht nur aus Sicht der Kostenträger, sondern auch aus derjenigen der Praxis.

Mit Sicherheit kann die MPA genau definierte Aufgaben des Hausarztes übernehmen, denn dies hat sie schon immer getan. Im Verlauf der immer stärkeren gesetzlichen Reglementierung der Hausarztpraxis (mittlerweile über 1200 Seiten Gesetze, Verordnungen, Richtlinien usw.) war es die MPA, die innerhalb des Praxisteams für die Umsetzung der Vorschriften sorgte und sorgt. Das nötige Fachwissen dazu erhielt sie im Rahmen ihrer Berufsausbildung, deren Inhalte von Ärzten und MPA in gemeinsamer Arbeit stetig an die neuen Erfordernisse der ambulanten Medizin angepasst wurden. Jeder, der sich im Mikrokosmos einer Grundversorgerpraxis ein wenig auskennt, weiss, dass die MPA sehr verantwortungsvolle Tätigkeiten ausübt, und dass ein Hausarzt nicht mehr auf seine MPA verzichten kann. Es ist ein Verdienst des Praxisteams und nicht nur des Arztes oder der Ärztin, dass es die Hausarztpraxis im heutigen Sinne überhaupt gibt. Dass dies auch so bleiben kann, dafür werden Ärzte und Medizinische Praxisassistentinnen erneut gemeinsam sorgen - diesmal durch die Aufwertung des MPA-Berufes in Form einer Weiterbildung auf Tertiärstufe mit eidgenössischem Fachausweis und der Delegation von zusätzlichen Aufgaben und Kompetenzen - auch ärztlichen.

Es ist an der Zeit, dass unsere Gesundheitspolitik und die Fachgremien unseres Gesundheitswesens die Existenz der MPA und des Projektes «Medizinische Praxiskoordinatorin» zur Kenntnis nimmt - «darum herumkommen werden sie nicht», wie die Arbeitsgruppe der SAMW im umgekehrten Zusammenhang so schön sagt.

\section{Literatur}

1 Arbeitsgruppe SAMW. Die zukünftigen Berufsbilder von ÄrztInnen und Pflegenden in der ambulanten und klinischen Praxis. Schweizerische Akademie der Medizinischen Wissenschaften; 2011.

2 Bourgeault L, Kuhlmann E, Neiterman E, Wrede S. Wie kann ein optimaler Qualifikationsmix effektiv verwirklicht werden - und warum? Weltgesundheitsorganisation im Namen des Europäischen Observatoriums für Gesundheitssysteme und Gesundheitspolitik; 2008.

- Arbeitsgruppe «Berufsbilder» der SAMW. Die zukünftigen Berufsbilder von Ärztinnen/Ärzten und Pflegenden in der ambulanten und klinischen Praxis. Schweiz. Ärztezeitung. 2007; 88(46):1942-52.

- Rosemann T, Schalch E, Birbaum B, Zanoni U. Grundversorgung: Der Weg aus der Krise führt auch über die MPA. Schweiz Ärztezeitung. 2010;91(28):1081-3.

- Gut C. Nurse practitioners in der medizinischen Grundversorgung der Schweiz? Schweiz Ärztezeitung. 2007;88(51):2170-3. 